\title{
Phase II Study of Irinotecan plus S-1 in Treatment of Advanced Gastric Cancer*
}

\author{
Hideki Bou $^{1 \#}$, Akira Tokunaga ${ }^{1}$, Hideyuki Suzuki ${ }^{1}$, Nobuo Murata², Yasuyuki Sugiyama², \\ Naoto Fukuda ${ }^{2}$, Masahiro Ishimaru ${ }^{3}$, Hiroyuki Suzuki ${ }^{3}$
}

${ }^{1}$ Institute of Gastroenterology, Nippon Medical School Musashi Kosugi Hospital, Kawasaki, Japan; ${ }^{2}$ Department of Surgery, University Hospital of Mizonokuchi, Teikyo University School of Medicine, Kawasaki, Japan; ${ }^{3}$ Department of Surgery, Kanto Rosai Hospital, Kawasaki, Japan.

Email: "bou@nms.ac.jp

Received December $28^{\text {th }}, 2012$; revised January $31^{\text {st }}, 2013$; accepted February $8^{\text {th }}, 2013$

Copyright (C) 2013 Hideki Bou et al. This is an open access article distributed under the Creative Commons Attribution License, which permits unrestricted use, distribution, and reproduction in any medium, provided the original work is properly cited.

\begin{abstract}
Objective: The efficacy and safety of irinotecan hydrochloride (CPT-11) plus oral fluoropyrimidine S-1 combination therapy in patients with previously untreated advanced gastric cancer was evaluated. Methods: The regimen comprised CPT-11 plus S-1: CPT-11, $60 \mathrm{mg} / \mathrm{m}^{2}$ (days 1, 15); S-1, $40-60 \mathrm{mg} /$ body twice daily (days $1-21$ ) followed by a 1-week rest, every 4 weeks. Primary endpoint was response rate. Secondary endpoints were tumor control rate, adverse events, relative dose intensity, and overall survival. Results: Twenty-five patients were enrolled; median age was 66 years. Response rate was $40 \%$ (95\% confidence interval, $21.1 \%$ - 61.3\%; complete response in 1; partial response in 9). Tumor control rate was $56.0 \%$, median survival time was 436 days and relative dose intensities were 0.83 for CPT- 11 and 0.85 for S- 1 . Incidence of grade 3 or greater neutropenia, anemia and diarrhea was $16 \%, 12 \%$, and $12 \%$, respectively. Conclusion: The present results indicate that CPT-11 plus S-1 offers lower treatment-related toxicity than regimens including cisplatin and is effective in patients with advanced gastric cancer.
\end{abstract}

Keywords: Advanced Gastric Cancer; Systemic Chemotherapy; Irinotecan; S-1

\section{Introduction}

Although chemotherapy has been reported to significantly prolong survival compared with best supportive care in the treatment of advanced or recurrent gastric cancer [1-3], the standard regimen remains to be established. In Japan, 5-fluorouracil (5-FU) is the most common choice in the treatment of advanced or recurrent gastric cancer. In 1992, the Japan Clinical Oncology Group (JCOG) conducted a study to compare monotherapy with 5-FU with 5-FU+cisplatin (CDDP) (FP) or UFT+mitomycin C (UFTM). Neither FP nor UFTM produced better therapeutic results than monotherapy with 5-FU [4]. This suggests limitations in exploring standard treatment based mainly on 5-FU, and therefore the advent of a new antitumor agent is awaited.

Irinotecan hydrochloride (CPT-11) is an antitumor agent developed in Japan. Its mechanism of action in-

\footnotetext{
"Conflict of Interest Statement: None of the authors have any conflicts of interest associated with this study

${ }^{\#}$ Corresponding author.
}

volves the inhibition of topoisomerase I (Topo I) [5], and it has been reported to be effective against advanced gastrointestinal cancer [6]. The response rate (RR) to monotherapy with CPT- 11 was $18.4 \%$ in eligible patients with advanced or recurrent gastric cancer. Approximately half of the patients developed reversible leucopenia. Clinical studies of combination therapy with CPT-11 and other chemotherapeutic agents have also shown promising results [7]. The RR after combination chemotherapy with CPT- 11 and CDDP has been reported to be between $42 \%$ and $59 \%$, and median survival time (MST) to be 365 days [8-10]. However, combination therapy with CDDP is highly toxic and has been reported to involve a high incidence of adverse events. Yano et al. [11] stated that CDDP should only be used by specialists who were familiar with treatment with antitumor agents.

An oral 5-FU derivative developed in Japan, S-1, is now available in capsule form together with tegafur (a prodrug of 5-FU), gimeracil (5-Chloro-2, 4-dihydroxypyridine) and otastat potassium. This combination is believed to enhance the antitumor effect of 5-FU by in- 
creasing its concentration and reducing gastrointestinal toxicity $[12,13]$. As the response rate to monotherapy with this drug is as high as $49 \%$ to $53.6 \%$ in patients with stomach cancer, it is now becoming the de facto standard treatment for advanced or recurrent gastric cancer $[14,15]$. The effects and therapeutic results of chemotherapy based on 5-FU, including S-1, are related to expression of thymidylate synthase (TS) [16]. Ichikawa et al. [17] showed that whereas monotherapy with S-1 produced a poor therapeutic effect in patients with high TS expression, S-1 in combination with CPT-11 was effective. Therefore, clinical studies of combination therapy with CPT11 and S-1 have been conducted in patients with advanced gastric cancer [18-21].

A number of studies have investigated the optimum dosage for combination therapy with CPT-11 and S-1. Therefore, this multicenter phase II focused on investigating the efficacy and safety of CPT-11 plus S-1 combination therapy in patients with advanced gastric cancer.

\section{Patients and Methods}

\subsection{Eligibility Criteria}

Patients with unresectable advanced or recurrent gastric cancer were enrolled. The patients were required to satisfy the following eligibility criteria: histologically confirmed diagnosis of gastric cancer with measurable lesion; age of 20 to 75 years; Eastern Cooperative Oncology Group (ECOG) performance status of $0-1$; no prior chemotherapy regimen including CPT-11 or S-1; leukocyte count of $4000-12,000 / \mathrm{mm}^{3}$, platelet count of $>100,000$ $\mathrm{mm}^{3}$, and hemoglobin $>9.5 \mathrm{~g} / \mathrm{dl}$; aspartate aminotransferase and alanine aminotransferase $<100 \mathrm{IU} / 1$ and total bilirubin $<1.5 \mathrm{mg} / \mathrm{dl}$; creatinine level of $<1.2 \mathrm{mg} / \mathrm{dl}$; electrocardiogram findings, normal. Written informed consent was required from all patients prior to participation in the study. Patients with any of the following conditions were excluded: severe, co-existing medical illness (including intestinal paresis or ileus, interstitial pneumonia, pulmonary fibrosis, and poorly controlled diabetes mellitus); severe psychiatric disturbances. This study was conducted with the approval of the ethics committee of each participating institution.

\subsection{Treatment Schedule}

Irinotecan was initially given at a dose of $60 \mathrm{mg} / \mathrm{m}^{2}$ over $90 \mathrm{~min}$ by intravenous infusion on days 1 and 15 (Figure 1). S-1 was administered orally according to body surface area twice daily (40 - $60 \mathrm{mg} /$ body twice daily, Table 1) for 3 consecutive weeks. This treatment was repeated every 4 weeks until disease progression, refusal by the patient, or unacceptable adverse reaction.

Prior to chemotherapy, patients received antiemetics comprising $5-\mathrm{HT}_{3}$ receptor antagonists and steroids. Epi- sodes of diarrhea were treated with loperamide hydrochloride as required.

The following dose adjustments were permitted: if grade 4 hematologic or grade 2 non-hematologic toxicity occurred, the dose of CPT-11 was reduced to $40 \mathrm{mg} / \mathrm{m}^{2}$ and treatment continued; if serum creatinine level reached 1.2 to $1.5 \mathrm{mg} / \mathrm{dl}$, the dose of $\mathrm{S}-1$ was reduced by $16.7 \%-32.5 \%$ (Table 2) and treatment continued.

\section{Evaluation}

The primary endpoint was RR; the secondary endpoints were tumor control rate, adverse events, relative dose intensity, and overall survival.

Tumor response was evaluated based on change in the size of measurable lesions and assessment of evaluable lesions. Measurable lesions and evaluable lesions were defined and efficacy evaluated in accordance with the Japanese Criteria for Evaluating the Efficacy of Chemotherapy and Radiation Therapy in the Treatment of Gastric Cancer [22]. In brief, complete response (CR) was

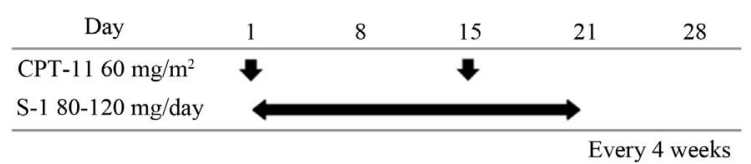

Figure 1. Treatment schedule.

Table 1. Criterial dose of S-1.

\begin{tabular}{cc}
\hline Body surface & Dose (as tegafur) \\
\hline$<1.25 \mathrm{~m}^{2}$ & $40 \mathrm{mg} /$ once \\
$1.25 \mathrm{~m}^{2}-1.5 \mathrm{~m}^{2}$ & $50 \mathrm{mg} /$ once \\
$\geq 1.5 \mathrm{~m}^{2}$ & $60 \mathrm{mg} /$ once \\
\hline
\end{tabular}

Table 2. Criteria for dose reduction.

\begin{tabular}{|c|c|c|c|}
\hline & \multirow[b]{2}{*}{ Grade } & \multicolumn{2}{|c|}{ Dose level } \\
\hline & & $\begin{array}{l}\text { CPT-11 } \\
\left(\mathrm{mg} / \mathrm{m}^{2}\right)\end{array}$ & $\begin{array}{c}\mathrm{S}-1 \\
\text { (mg/once) }\end{array}$ \\
\hline WBC & $<1000 / \mathrm{mm}^{3}$ & $60 \rightarrow 40$ & - \\
\hline Platelet & $<50,000 / \mathrm{mm}^{3}$ & $60 \rightarrow 40$ & - \\
\hline \multirow[t]{2}{*}{ Diarrhea } & $\begin{array}{l}\text { NCI-CTC } \geq \text { grade } 2 \\
\text { or watery diarrhea }\end{array}$ & $60 \rightarrow 40$ & - \\
\hline & & & $40 \rightarrow 25$ \\
\hline \multirow[t]{2}{*}{ Serum creatinine } & $1.2-1.5 \mathrm{mg} / \mathrm{dl}$ & - & $50 \rightarrow 40$ \\
\hline & & & $60 \rightarrow 50$ \\
\hline $\begin{array}{l}\text { Other adverse } \\
\text { events }\end{array}$ & $\begin{array}{l}\text { Greater than } \\
\text { grade } 3\end{array}$ & $60 \rightarrow 40$ & - \\
\hline
\end{tabular}

*Excluding abnormal laboratory findings, nausea, appetite loss and general fatigue. 
defined as the disappearance of all evidence of the tumor for at least 4 weeks. Partial response (PR) was defined as a $50 \%$ or greater reduction in the sum of the products of the perpendicular diameters of all measurable lesions for at least 4 weeks without any evidence of new lesions or the progression of any existing lesions. Stable disease (SD) was defined as a less than $50 \%$ reduction or less than $25 \%$ increase in the sum of the products of the perpendicular diameters of all lesions for at least 4 weeks without any evidence of new lesions or the progression of any existing lesions. Progressive disease was defined as a $>25 \%$ increase in one or more lesions or the appearance of new lesions. Tumor measurements were performed every 4 weeks using computed tomography, plain chest X-ray films, upper gastrointestinal endoscopy, and ultrasonography. Primary tumors were classified as follows based on X-ray and endoscopic findings: measureable, not measurable but evaluable, or diffused infiltration.

The NCI-CTC ver. 2.0 was used to evaluate adverse events. An independent committee reviewed the eligibility and suitability for assessment of the subjects and response to treatment.

\section{Results}

\subsection{Patient Characteristics}

Twenty-five patients ( 23 men, 2 women) were enrolled in this study between November 2004 and August 2008. The clinical characteristics of the patients are shown in Table 3. All patients met the entry criteria and were included in the analysis. Three patients $(3 \mathrm{men})$ had received prior chemotherapy (doxifluridine (5'-DFUR) was used in 2 and UFT+Paclitaxel (PTX) in 1). The median age of the patients was 66 years (range, 47 - 78 years). Histologically, 11 patients had poorly differentiated adenocarcinoma, 10 had tubular adenocarcinoma, 2 had signet-ring cell carcinoma, 1 had papillary adenocarcinoma, and 1 was unknown. Performance status was 0 in 23 patients and 1 in 2 patients.

\subsection{Tumor Response and Survival}

Among 25 patients with evaluable lesions, CR was observed in 1 patient and PR in 9. The overall RR was $40 \%$ (95\% confidence interval, 21.1\% - 61.3\%) in Table 4. The tumor control rate (SD or better response) was $56.0 \%$ (14/25). The RR according to site was $41.7 \%(5 / 12)$ for abdominal lymph node metastases and $27.3 \%(3 / 11)$ for liver metastases. Median survival time was 436 days ( $95 \%$ confidence interval, $61-212$ days) in the 25 patients (Figure 2). Median number of treatment cycles given was 4 (range, 0.5 - 19). Patients were taken out of the study due to worsening of primary disease (10 patients), adverse events ( 8 patients), refusal of treatment (1 patient), operation ( 2 patients), or other (4 patients). Relative dose intensities were 0.83 for CPT-11 and 0.85 for $\mathrm{S}-1$.

Table 3. Patient characteristics.

\begin{tabular}{|c|c|c|}
\hline & & $\mathrm{N}=25$ \\
\hline \multicolumn{3}{|c|}{ Sex } \\
\hline & Male & 23 \\
\hline & Female & 2 \\
\hline
\end{tabular}

Age (years)

Median (range) $\quad 66(47-78)$

Performance status

$\begin{array}{ll}0 & 23 \\ 1 & 2\end{array}$

Prior chemotherapy

\begin{tabular}{|c|c|}
\hline No & \\
\hline Yes & \\
\hline & 5'-DFUR \\
\hline & UFT/PTX \\
\hline
\end{tabular}

Primary tumor

$\begin{array}{cc}\text { No } & 5 \\ \text { Yes } & 20\end{array}$

Histology

$\begin{array}{rc}\text { Intestinal } & 11 \\ \text { Diffuse } & 13 \\ \text { Other } & 1\end{array}$

Metastatic site

$\begin{array}{rc}\text { Liver } & 11 \\ \text { Lymph node } & 12 \\ \text { Other } & 9\end{array}$

Nunber of metastatic site

$\begin{array}{rc}0 & 3 \\ 1 & 16 \\ 2 \text { or more } & 6\end{array}$

Table 4. Response rate (RR).

\begin{tabular}{ccccccc}
\hline & $\mathrm{CR}$ & $\mathrm{PR}$ & $\mathrm{SD}$ & $\mathrm{PD}$ & $\mathrm{NE}^{*}$ & $\mathrm{RR}(\%)$ \\
\hline Overall $(\mathrm{n}=25)$ & 1 & 9 & 4 & 5 & 6 & 40 \\
& & & & & & \\
Intestinal type & 1 & 6 & 1 & 1 & 2 & 64 \\
Diffuse type & 0 & 3 & 3 & 3 & 4 & 23 \\
\hline
\end{tabular}

"NE: not evaluable. CR: complete response; PR: partial response; SD: stable disease; PD: progressive disease. 


\subsection{Safety}

Adverse events among the 25 patients are shown in Table 5 . The main grade $3 / 4$ hematological toxicities observed were leucopenia $(12 \%)$, neutropenia $(16 \%)$, and anemia (12\%). The grade $3 / 4$ non-hematological toxicity was diarrhea $(12 \%)$. Combination therapy was tolerable and mild. No treatment-related deaths occurred.

\section{Discussion}

Mainstream chemotherapy for advanced, recurrent gastric cancer involves multiple agents, which provide a high anti-tumor effect and survival benefit. In the US, 5-FU + ADR + MTX (FAMTX) used to be the standard therapy in such cases. In recent years, however, epirubicin + CDDP + 5-FU (ECF) therapy has become a standard option in such patients following the publication of

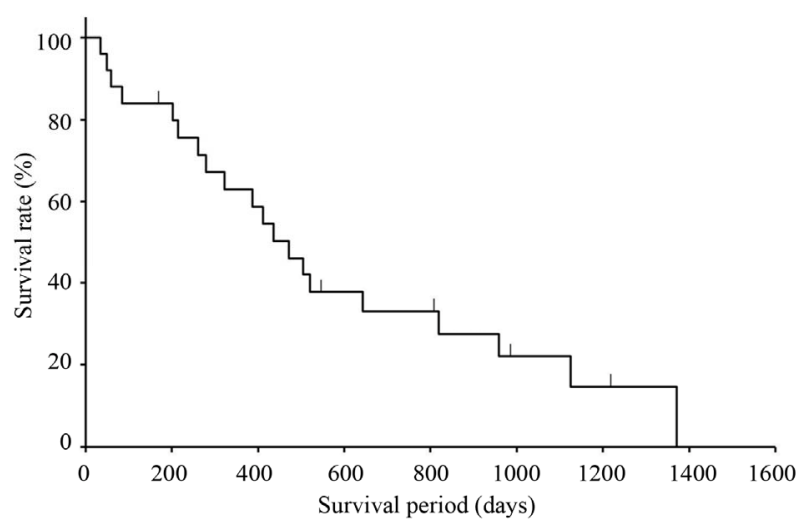

Figure 2. Overall survival.

Table 5. Toxicity.

\begin{tabular}{ccccccc}
\hline & \multicolumn{3}{c}{ Grade } & All Grades & Grade 3/4 \\
\cline { 2 - 7 } & 1 & 2 & 3 & 4 & $(\%)$ & $(\%)$
\end{tabular}

Hematological

\begin{tabular}{rcccccc} 
Leucopenia & 8 & 9 & 3 & 0 & 80 & 12 \\
Neutropenia & 7 & 5 & 4 & 0 & 64 & 16 \\
Anemia & 8 & 10 & 3 & 0 & 84 & 12 \\
Thrombocytopenia & 2 & 1 & 1 & 0 & 16 & 4 \\
Non-hematological & & & & & & \\
Nausea & 5 & 1 & 1 & 0 & 28 & 4 \\
Vomiting & 3 & 1 & 0 & 0 & 16 & 0 \\
Anorexia & 7 & 2 & 2 & 0 & 44 & 8 \\
Stomatitis & 3 & 2 & 1 & 0 & 24 & 4 \\
Diarrhea & 5 & 3 & 3 & 0 & 44 & 12 \\
Enteritis & 0 & 0 & 1 & 0 & 4 & 4 \\
Fever & 3 & 1 & 0 & 0 & 16 & 0 \\
\hline
\end{tabular}

two studies: the US study V325, in which 5-FU/CDDP (CF) was compared with CF plus docetaxel (DCF), and a European study, in which FAMTX was compared with ECF. These triplet regimens can exert a high anti-tumor effect, but may also be accompanied by severe toxicity.

In Japan, continuous intravenous infusion of 5-FU has been used as a reference arm in trials, and various doublet regimens have been studied. Recent phase III studies such as JCOG9912 [23] and SPIRITS [24] have changed the use of each of these agents. The JCOG9912 study demonstrated the non-inferiority of $\mathrm{S}-1$ over continuous 5-FU infusion, but not the superiority of CPT-11/CDDP therapy. CPT-11/CDDP was shown to be superior to continuous 5-FU infusion, with a significant improvement in progression-free survival (4.8 months vs. 2.9 months, $\mathrm{p}<0.001$ ), but no significant difference in MST (12.3 months vs. 10.8 months, $\mathrm{p}=0.055)$. Consequently, in light of its milder toxic effects, S-1 became a key drug in Japan. Around the same time, the SPIRITS study evaluated the efficacy of CDDP added to S-1. Progressionfree survival was 4.0 months vs. 6.0 months $(p<0.0001)$, demonstrating the efficacy of this add-on therapy. Thus, Japanese guidelines currently recommend S-1/CDDP for first-line treatment for advanced, recurrent gastric cancer. Meanwhile, the phase III GC0301 study [25] investigated the efficacy of CPT-11 when added to S-1. A significant difference was observed in RR $(26.9 \%$ vs. $41.5 \%, \mathrm{p}=$ $0.035)$, but not in survival (10.5 months vs. 12.8 months, $\mathrm{p}=0.233$ ), which was the primary endpoint of that study. CPT-11/S-1 therapy is not presently recommended as first-line therapy. However, CPT-11 remains an effective second-line agent.

At the time of planning the present study, both the JCOG9912 and SPIRITS studies were still in progress, and the most recent JCOG9205 report did not show an additional effect of CDDP. Therefore, we decided to investigate CPT-11/S-1 therapy, taking into account that hydration requires short-term hospitalization. We designed a regimen aimed at lower toxicity and higher treatment continuity based on the CPT-11/S-1 results reported in the OGSG002 trial [26]. We left the dose intensity unaltered, but reduced the amount of each dose of CPT11 , which is known to exert severe toxic effects, even when administered at a low dose. The results suggest a new treatment option.

The results of the present study showed a good survival outcome (436 days), although the overall RR (40\%) was relatively low compared with that in other phase II studies. Interestingly, the treatment effect varied depending on histological type, with RR (Table 4), MST (959 days, 279 days), and time-to-treatment failure (248 days, 102 days) being better in patients with differentiated carcinoma. These results were comparable with those in the OGSG002 study (overall RR, 47.8\%; intestinal RR, 
$60.0 \%$; diffuse RR, $38.5 \%$ ); and in a retrospective study [10] of CPT-11/CDDP therapy, differentiated carcinoma was shown to have a significantly greater survival time (differentiated, 472 days vs. undifferentiated, 291 days, $\mathrm{p}=$ 0.0115). Thymidylate synthase activity may explain this. Ichikawa et al. confirmed higher TS activity in patients with differentiated gastric cancer, as well as a lower effect of S-1 monotherapy. They also reported that the addition of CPT-11 down-regulates TS activity, so that a therapeutic effect could be expected, even if TS activity was high. Thus, CPT-11 may exert a higher treatment effect, particularly in differentiated gastric cancer. This is consistent with the findings of Katsube et al. [27].

Sub-analyses of the SPIRITS and JCOG9912 studies, both of which involved CDDP as a drug of interest, showed superior outcomes in diffuse-type gastric cancer, suggesting that the treatment effect of CDDP is markedly higher in this type. The GC0301 study also indicated that combination therapy with CPT-11 may reduce risks in diffuse-type gastric cancer. However, Japanese phase III studies have not taken into account the presence or absence of a target (measurable) lesion, and the patient characteristics have been substantially different from ours, with eligible patients showing an RR of approximately $60 \%$ compared with the overall patient population (SPIRIT and GC0301 studies). Taking this into account, we cannot exclude the possibility that other factors may be involved. Furthermore, the JCOG9912 study showed that CPT-11/CDDP therapy yielded a significantly higher survival benefit than 5-FU in patients with target lesions, while a statistically significant improvement in survival time was observed for S-1 monotherapy in those with nontarget lesions. In addition, combination with CDDP significantly improved overall survival in patients with peritoneal dissemination and/or patients without target lesions in the SPIRITS trial. Therefore, CPT-11 is considered to have a high therapeutic effect, even for target lesions, and it may be possible to select treatment based on pathological condition in patients whose gastric cancers have hetero-type tumor characteristics.

The results of the present study, which have been confirmed to be reproducible, suggest that CPT-11/S-1 therapy has lower treatment-related toxicity than regimens including CDDP and a greater effect in differentiated carcinoma. Although sufficient efficacy was not established in phase III studies in which first-line regimens were investigated, this CPT-11-containing treatment strategy may well exert a therapeutic effect in patients with differentiated-type tumors and/or target lesions, warranting further studies focusing on such patient populations.

\section{REFERENCES}

[1] A. M. Murad, F. F. Santiago, A. Petroianu, et al., "Modi- fied Therapy with 5-Fluorouracil, Doxorubicin, and Methotrexate in Advanced Gastric Cancer," Cancer, Vol. 71, No. 1, 1993, pp. 37-41.

doi:10.1002/1097-0142(19930701)72:1<37::AID-CNCR2 $\underline{820720109>3.0 . \mathrm{CO} ; 2-\mathrm{P}}$

[2] B. Glimelius, K. Hoffman, U. Haglund, O. Nyren and P. O. Sjoden, "Initial or Delayed Chemotherapy with Best Supportive Care in Advanced Gastric Cancer," Annals of Oncology, Vol. 5, No. 2, 1994, pp. 189-190.

[3] S. Pyrhonen, T. Kuitunen, P. Nyandoto and M. Kouri, "Randomized Comparison of Fluorouracil, Epidoxorubicin and Methotrexate (FEMTX) plus Supportive Care with Supportive Care Alone in Patients with Non-Resectable Gastric Cancer," British Journal of Cancer, Vol. 71, No. 3, 1995, pp. 587-591. doi:10.1038/bjc.1995.114

[4] A. Ohtsu, Y. Shimada, K. Shirao, et al., "Randomized Phase 3 Trial of Fluorouracil Alone versus Fluorouracil plus Cisplatin versus Uracil and Tegafur plus Mitomycin in Patients with Unresectable, Advanced Gastric Cancer: The Japan Clinical Oncology Group Study (JCOG9205)," Journal of Clinical Oncology, Vol. 21, No. 1, 2003, pp. 54-59. doi:10.1200/JCO.2003.04.130

[5] Y. Kawano, M. Aonuma, Y. Hirota, H. Kuga, K. Sato, et al., "Intracellular Roles of SN-38, a Metabolite of the Camptothecin Derivative CPT-11, in the Antitumor Effect of CPT-11," Cancer Research, Vol. 51, No. 16, 1991, pp. 4187-4191.

[6] Y. Sakata, I. Nakao, K. Futatsuki, et al., "An Early Phase II Trial of CPT-11 in Patients with Advanced Gastrointestinal Cancer," Journal of Japan Society for Cancer Therapy, Vol. 27, No. 12, 1992, pp. 2028-2035.

[7] K. Futatsuki, A. Wakui, I. Nakao, et al., "Late Phase II Study of Irinotecan Hydrochloride (CPT-11) in Advanced Gastric Cancer. CPT-11 Gastrointestinal Cancer Study Group," Japanese Journal of Cancer and Chemotherapy, Vol. 21, No. 7, 1994, pp. 1033-1038.

[8] K. Shirao, Y. Shimada, H. Kondo, et al., "Phase I-II Study of Irinotecan Hydrochloride Combined with Cisplatin in Patients with Advanced Gastric Cancer," Journal of Clinical Oncology, Vol. 15, No. 3, 1997, pp. 921-927

[9] N. Boku, A. Ohtsu, Y. Shimada, et al., "Phase II Study of a Combination of Irinotecan and Cisplatin against Metastatic Gastric Cancer," Journal of Clinical Oncology, Vol. 17, No. 1, 1999, pp. 319-323.

[10] M. Yoshida, N. Boku, A. Ohtsu, et al., "Combination Chemotherapy of Irinotecan plus Cisplatin for Advanced Gastric Cancer: Efficacy and Feasibility in Clinical Practice," Gastric Cancer, Vol. 4, No. 3, 2001, pp. 144-149. doi:10.1007/PL00011737

[11] T. Yano and A. Ohtsu, "Evaluation of Newly Developed Agents for Gastric Cancer," Japanese Journal of Cancer Clinics, Vol. 49, No. 7, 2003, pp. 583-588.

[12] T. Fukushima, H. Satake, J. Uchida, et al., "Preclinical Antitumor Efficacy of S-1: A New Oral Formulation of 5-Fluorouracil on Human Tumor Xenografts," International Journal of Oncology, Vol. 13, No. 4, 1998, pp. 693698.

[13] T. Shirasaka, K. Nakano, T. Takechi, et al., "Antitumor Activity of $1 \mathrm{M}$ Tegafur-0.4 M 5-Chloro-2,4-Dihydroxy- 
pyridine-1 M Potassium Oxonate (S-1) against Human Colon Carcinoma Orthotopically Implanted into Nude Rats," Cancer Research, Vol. 56, No. 11, 1996, pp. 2602-2606.

[14] K. Sugimachi, Y. Maehara, N. Horikoshi, et al., "An Early Phase II Study of Oral S-1, a Newly Developed 5Fluorouracil Derivative for Advanced and Recurrent Gastrointestinal Cancers, the S-1 Gastrointestinal Cancer Study Group," Oncology, Vol. 57, No. 3, 1999, pp. 202-210. doi:10.1159/000012032

[15] Y. Sakata, A. Ohtsu, N. Horikoshi, et al., "Late Phase 2 Study of Novel Oral Fluoropyrimidine Anticancer Drug S-1 in Advanced Gastric Cancer Patients," European Journal of Cancer, Vol. 34, No. 11, 1998, pp. 1715-1720. doi:10.1016/S0959-8049(98)00211-1

[16] H. J. Lenz, C. G. Leichman, K. D. Danenberg, et al., "Thymidylate Synthase mRNA Level in Adenocarcinoma of the Stomach: A Predictor for Primary Tumor Response and Overall Survival," Journal of Clinical Oncology, Vol. 14, No. 1, 1996, pp. 176-182.

[17] W. Ichikawa, T. Takahashi, K. Suto, et al., "Thymidylate Synthase Predictive Power Is Overcome by Irinotecan Combination Therapy with S-1 for Gastric Cancer," British Journal of Cancer, Vol. 91, No. 7, 2004, pp. 12451250. doi:10.1038/sj.bjc.6602139

[18] H. Takiuchi, H. Nakahara, T. Tsujinaka, et al., "Phase I Study of S-1 Combined with Irinotecan (CPT-11) in Patients with Advanced Gastric Cancer (OGSG 0002)," Japanese Journal of Clinical Oncology, Vol. 35, No. 9, 2005, pp. 520-525. doi:10.1093/ijco/hyi148

[19] K. Fujitani, H. Takiuchi, H. Marahara, et al., "A Phase I/II Study of CPT-11 Plus S-1 in Patients with Advanced Gastric Cancer," Japanese Journal of Cancer Clinics, Vol. 49, No. 7, 2003, pp. 605-609.

[20] Y. Komatsu, H. Takeda. M. Takei, et al., "A Phase I and PK Study of S-1 and Irinotecan (CPT-11) in Patients with Advanced Gastric Cancer (AGC)," Proceedings of the 38th
American Society of Clinical Oncology, Orlando, 18-21 May 2002, p. 171.

[21] Y. Yamada, H. Yasui, A. Goto, et al., "Phase I Study of Irinotecan and S-1 Combination Therapy in Patients with Metastatic Gastric Cancer," International Journal of Clinical Oncology, Vol. 8, No. 6, 2003, pp. 374-380. doi:10.1007/s10147-003-0359-z

[22] Japanese Research Society for Gastric Cancer, "Japanese Classification of Gastric Carcinoma," Kanehara \& Co., Ltd., Tokyo, 1995.

[23] N. Boku, S. Yamamoto, H. Fukuda, et al., "Fluorouracil versus Combination of Irinotecan plus Cisplatin versus S-1 in Metastatic Gastric Cancer: A Randomised Phase 3 Study," The Lancet Oncology, Vol. 10, No. 11, 2009, pp. 1063-1069. doi:10.1016/S1470-2045(09)70259-1

[24] W. Koizumi, H. Narahara, T. Hara, et al., "S-1 plus Cisplatin versus S-1 Alone for First-Line Treatment of Advanced Gastric Cancer (SPIRITS Trial): A Phase III Trial," The Lancet Oncology, Vol. 9, No. 3, 2008, pp. 215-221. doi:10.1016/S1470-2045(08)70035-4

[25] H. Narahara, H. Iishi, H. Imamura, et al., "Randomized Phase III Study Comparing the Efficacy and Safety of Irinotecan plus S-1 with S-1 Alone as First-Line Treatment for Advanced Gastric Cancer (Study GC0301/TOP-002)," Gastric Cancer, Vol. 14, No. 1, 2011, pp. 72-80. doi:10.1007/s10120-011-0009-5

[26] N. Uedo, H. Narahara, R. Ishihara, et al., "Phase II Study of a Combination of Irinotecan and S-1 in Patients with Advanced Gastric Cancer (OGSG0002)," Oncology, Vol. 73, No. 1-2, 2007, pp. 65-71. doi:10.1159/000120630

[27] T. Katsube, K. Ogawa, W. Ichikawa, et al., "Phase I/II Study of Irinotecan (CPT-11) and S-1 in the Treatment of Advanced Gastric Cancer," Anticancer Drugs, Vol. 18, No. 5, 2007, pp. 605-610. doi:10.1097/CAD.0b013e3280262460 